(2) Open Access Full Text Article

\title{
Support for stroke patients in resumption of driving: patient survey and driving simulator trial
}

This article was published in the following Dove Press journal:

International Journal of General Medicine

3 March 2011

Number of times this article has been viewed

\section{Masahito Hitosugi' \\ Itaru Takehara ${ }^{2}$ \\ Shu Watanabe ${ }^{3}$ \\ Yasufumi Hayashi ${ }^{2}$ \\ Shogo Tokudome'}

'Dokkyo Medical University School of Medicine, Tochigi; ${ }^{2}$ Tokyo Metropolitan Rehabilitation Hospital, Tokyo; ${ }^{3}$ Tokyo Metropolitan University, Tokyo, Japan
Correspondence: Masahito Hitosugi Department of Legal Medicine, Dokkyo Medical University School of Medicine, 880 Kita-Kobayashi, Mibu,

Tochigi 32I-0293, Japan

$\mathrm{Tel}+8 \mid 282872135$

Fax +81282867678

Email hitosugi@dokkyomed.ac.jp
Background: Encouragement of stroke patients to resume driving is important to promote their reintegration into the community. Limited rehabilitation has been performed in this regard, owing to lack of specific knowledge on the part of medical staff. To establish an effective support program for stroke patients who wish to resume driving, we propose comprehensive training by medical staff using a driving simulator.

Methods: A survey of stroke patients admitted to the Tokyo Metropolitan Rehabilitation Hospital was first performed. A questionnaire was sent to 525 patients. Of 218 responses, the answers of 118 patients who had been driving before their stroke were analyzed. More than $80 \%$ of stroke patients did not obtain enough information about resuming driving during their hospital stay, and $38.1 \%$ of patients would have liked to have had driving training with a simulator. From these results, we set out to determine the effect of driving training using a realistic and technically advanced driving simulator. Twenty-four stroke patients and 20 healthy controls were included in the study.

Results: Repeat training with the simulator resulted in an increased ability to perform braking and an improvement in driving ability. The majority of stroke patients who had the mental and physical ability to drive a car were likely to be assessed as being able to resume driving as a result of the training program.

Conclusion: This study indicates that comprehensive support by medical staff and provision of adequate information about resumption of driving and the opportunity for training on a driving simulator are likely to aid resumption of driving by stroke patients, thus enhancing their rehabilitation and social reintegration.

Keywords: automobile driving, rehabilitation, stroke, support, training

\section{Introduction}

Patients recovering from stroke often want to return to driving in order to resume activities of everyday life. Although the ability to drive may be reduced in many patients, medical staff should support patients in this regard because it is an important element of their ability of participation in society. ${ }^{1,2}$ However, driving a vehicle is a complex task that requires a combination of motor, perceptual, visual, sensory, and cognitive skills. ${ }^{3}$

Under Japanese law, permission to drive a vehicle is decided after an audit of the results of simple perspective and reaction tests and the findings of reports by physicians by the Public Safety Commission. Furthermore, this process is initiated voluntarily by the stroke patient. Thus, in Japan, there are no clear objective guidelines for deciding whether a stroke patient can drive a vehicle, nor is there a comprehensive support 
program to increase the opportunity of stroke patients to resume driving. Also, there is a lack of specific knowledge in the part of staff and a lack of specific equipment in rehabilitation facilities, resulting in little encouragement of resumption of driving. ${ }^{4}$ Therefore, although physical and/or neurophysiological impairment does not necessarily preclude driving, many patients with stroke have difficulty in resuming driving.

To establish an effective support program for stroke patients who wish to drive again, we first investigated the rate of driving resumption and the severity of physical and cognitive impairment in a cohort of stroke patients. A driving resumption support program using a driving simulator and comprehensive support by medical staff was then assessed.

\section{Methods and materials}

\section{Survey of stroke patients}

We initially undertook a survey of stroke patients admitted to Tokyo Metropolitan Rehabilitation Hospital between April 2006 and February 2008. Their driving habits before and after the stroke were determined. In addition, we asked whether the patients received information about resumption of driving during their hospital stay and what kind of support they felt would be useful.

We recruited participants for the driving training program from inpatients admitted to the Tokyo Metropolitan Rehabilitation Hospital between November 2008 and January 2010. The inclusion criteria for this program were a diagnosis of stroke, having been an active driver before suffering the stroke, and a wish to resume driving. Twenty-four patients met these criteria and were included in the study after giving their written informed consent. In addition, 20 healthy volunteers participated in the training program as controls. The study was approved by the Ethical Committee of the Tokyo Metropolitan Rehabilitation Hospital.

Physicians and physical therapists evaluated the physical and mental states of all participants using the Mini-Mental State Examination (MMSE) and Brunnstrom stage. The type and site of the stroke, as well as the time interval between stroke onset and participation in the program were recorded.

\section{Driving simulation}

The Honda driving simulator (Honda, Tokyo), an interactive, realistic, and technically advanced simulator, was used in this program (Figure 1). This machine was sited in the Tokyo Metropolitan Rehabilitation Hospital to observe the driving performance of the participants. The simulator consisted of an actual front body of an automatic sedan car and was equipped

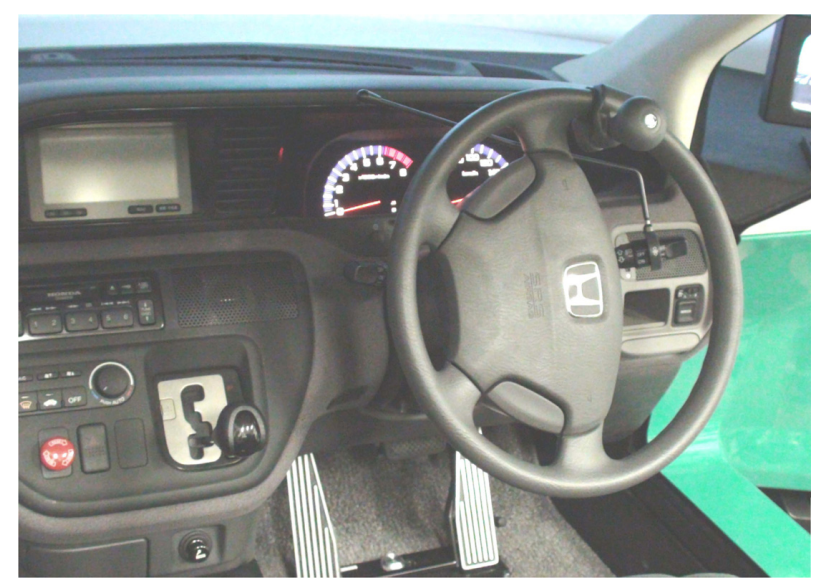

Figure I Modified driving simulator enabling patients with right or left hemiplegia to drive.

with three large screens with a field-of-view of 138 degrees. The simulator was modified for this program to allow use by patients with right hemiplegia. Adaptive aids, and a left-sided accelerator pedal, left-sided indicator stick, and steering spinner were incorporated into the simulator.

First, to become acquainted with the simulator, participants drove on a simulated seaside road at a speed of $30 \mathrm{~km} / \mathrm{h}$ or less. The route consisted of straight and curved roads with low traffic density and no unpredictable events. Next, the participants drove on a simulated straight road and were exposed to a hazard whereby they had to avoid a collision, ie, when driving at a constant speed of $40 \mathrm{~km} / \mathrm{h}$, a truck suddenly crossed the road $21.6 \mathrm{~m}$ in front of them; the participant was required to remove their foot from the accelerator and quickly step on the brake pedal to avoid a collision. We examined whether the participant performed the braking task, and measured their reaction speed and braking time. Reaction time was defined as the time between appearance of the truck and $10 \%$ of full-braking action. Braking time was defined as the duration between $10 \%$ of full-braking action and vehicle velocity reducing to less than $0.1 \mathrm{~km} / \mathrm{h}$. The participants could attempt the task up to three times.

\section{Statistical analysis}

The Chi-square test was used to compare the rates of successful braking to avoid collision between patient and control groups. The Mann-Whitney U test was used to compare the reaction and braking times between the two groups. The Wilcoxon test was used to compare the reaction time of the person between the first and second, and the second and third attempts at the task. Differences with a $P$ value less than 0.05 were considered to be statistically significant. The statistical analyses were conducted with Excel and Stacel. 


\section{Results}

\section{Survey of stroke patients}

The questionnaire was sent to 525 patients and, of the 216 responses, 118 patients (107 male, 11 female, mean age ( \pm standard deviation) $59.1 \pm 10.4$ years, had been driving a vehicle before their stroke. Of the 118 patients, only $42(35.6 \%)$ resumed driving after the stroke. Although some of the 118 patients did not wish to drive again, another $40(33.9 \%)$ patients still wished to be able to drive a vehicle again (Figure 2). Seventy-four of the 118 patients (62.7\%) answered "no" to the question: "Did you receive information about driving a vehicle during your hospital-stay". Fifteen of the patients $(12.7 \%)$ received adequate information and 22 patients (18.6\%) did not receive enough information during their hospital stay. Nearly half of the patients wanted guidance or a response to questions about driving resumption, and 45 (38.1\%) patients expressed a wish for car driving training using a simulator.

\section{Driving simulator training program}

Twenty-four stroke patients (20 male, four female; mean age $55.2 \pm 12.9$ years, range $37-76$ years) and 20 healthy volunteers ( 11 male, nine female; mean age $28.3 \pm 7.6$ years, range $21-49$ years) were included in this part of the study. No patient dropped out of the training program. Fourteen patients had suffered a cerebral infarction, nine a cerebral hemorrhage, and one a subarachnoid hemorrhage. Thirteen patients had right hemiplegia, seven had left hemiplegia, two had aphasia, one had ataxia, and one had an orientation disorder. The mean Brunnstrom stages were $5.3 \pm 1.1$ (range, 3-6) in the upper extremities; $5.3 \pm 1.2$ (range, $3-6$ ) in the hand; and 5.2 \pm 1.1 (range, 3-6) in the lower extremities. Mean MMSE was $27.9 \pm 2.3$ (range, 20-30). No patient had unilateral spatial neglect or abnormalities of visual depth perception. Throughout the training, we were able to communicate with the patients.

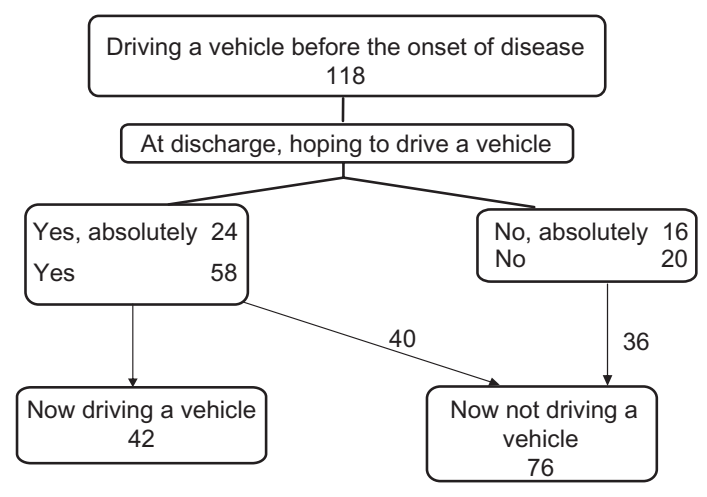

Figure 2 Number of patients driving before and after stroke.
The patients with left hemiplegia could operate the simulator with their right limbs. Ten of 13 patients with right hemiplegia and Brunnstrom stage 5 or 6 could operate the simulator with their right limbs. The remaining three patients with right hemiplegia and Brunnstrom stage 3 for the upper and lower right limbs operated the simulator with their left limbs.

On the first attempt at the braking task, eight of 24 patients (33.3\%) and 13 of 20 controls ( $65.0 \%$ ) were able to avoid a collision with the truck (Figure 3 ). The difference was statistically significant (Chi-square test, $P=0.03$ ). However, on the second or third attempt, the rates increased significantly to $72.7 \%$ and $86.4 \%$, respectively (Chi-square test, $P<0.001$ compared with that in the first trial). On the first attempt, the mean reaction time of the patients was 0.8 (range 0.5-1.2) seconds, which was significantly longer than that of healthy volunteers (0.7, range $0.5-1.3$ seconds, Mann-Whitney U test, $P=0.02$, Figure 4). However, mean reaction times were significantly shorter in all patients on the second and third attempts (0.7, range $0.5-0.9$ seconds and 0.6 , range $0.4-0.7$ seconds, respectively, Wilcoxon test, $P<0.001)$. For the patients who were able to avoid a collision, braking time was compared with that of the controls. On the first attempt, the mean braking time of patients and controls was 1.5 (1.5-1.6) seconds and 1.5 (1.5-1.5) seconds, respectively. The braking time of the patients remained the same on the second and third attempts (Figure 5).

\section{Discussion}

An important goal of this research was to increase the opportunity for stroke patients to resume driving. We first identified that medical staff did not provide sufficient information to stroke patients about resumption of driving. A recent report suggested that more than half of occupational therapists failed to discuss driving with their patients. ${ }^{5}$ Therefore, not only physicians, but also other staff (including nurses, occupational

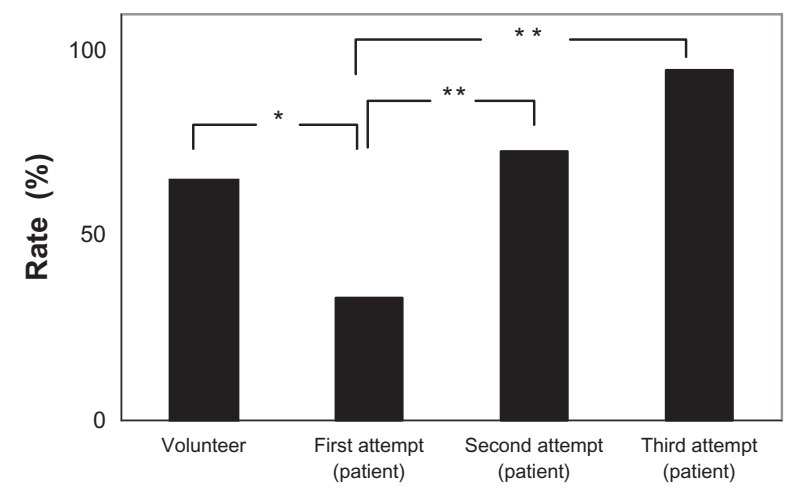

Figure 3 Rate of success in the braking task. 


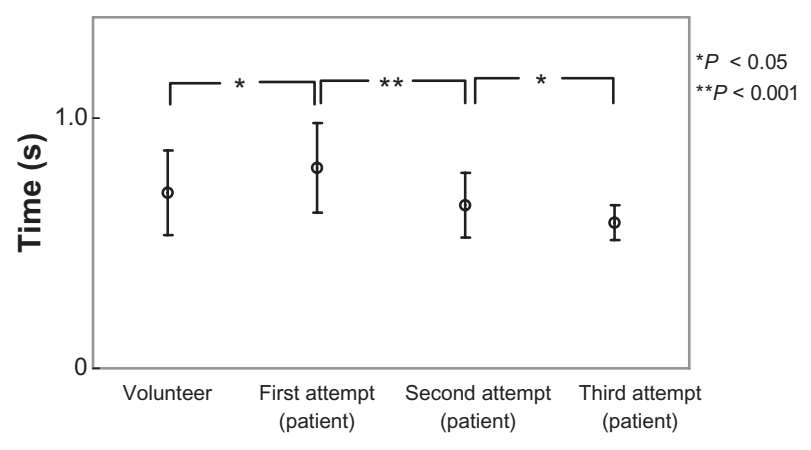

Figure 4 Reaction times in the braking task (symbol: mean value, error bar: \pm standard deviation).

therapists, physical therapists) should understand the process by which stroke patients start driving a vehicle again and provide useful information to their patients. Generally, for patients with stroke, frequent and regular contact initiated around the time of discharge after a stroke is desirable to prevent depression, reduction in quality of life, decreased functional recovery, and increased social isolation. ${ }^{6}$ Therefore, adequate information needs to be provided to the patient both before and after hospital discharge.

To maintain safety, the driving ability of a stroke patient needs to be estimated accurately. Complex cognitive, decisionmaking, and physical abilities are necessary for safe driving. Therefore, training and evaluation in the driving environment are needed. More than one third of our patients who had been driving a vehicle wished to have driving training with a simulator. A driving simulator can serve as a dynamic assessment and training tool that can be modified to improve specific physical, cognitive, and perceptual performance skills within the broader context of driving and mobility within the community. ${ }^{7}$

A simulator can improve driving skills by allowing patients to practice compensatory driving strategies in a less costly and risk-free environment. Simulator systems vary in their ability

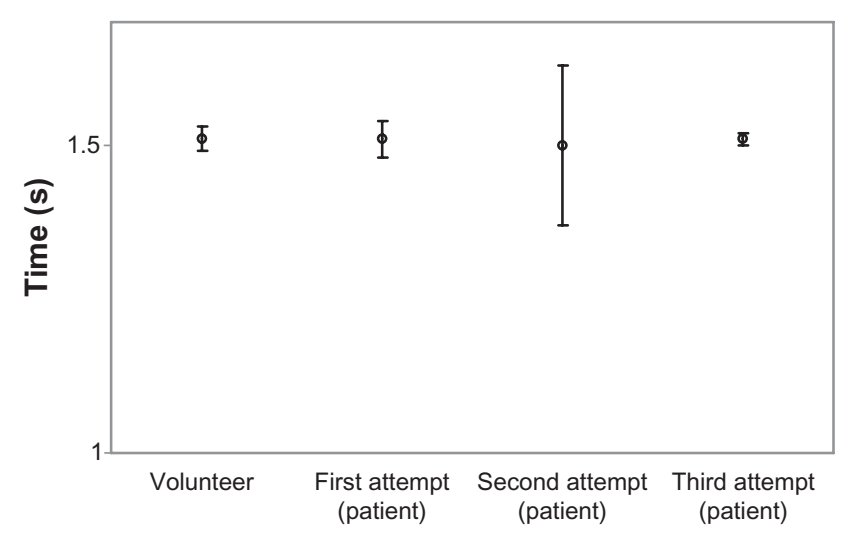

Figure 5 Braking times of subjects who carried out the braking task (symbol: mean value, error bar: \pm standard deviation). to simulate reality. The simulator used in this study had a wide screen with images that changed in response to the driver's input and had the same front body as a sedan-type car. Therefore, this simulator was able to provide a realistic driving environment. In addition, the driving simulator was modified so that patients with right or left hemiplegia could drive it. Because the driving simulator also offered a no-risk experience, it enabled patients who were anxious about any driving issue to test their driving ability in a safe environment that could help to reinforce that driving was a viable and worthwhile goal for that individual. Although driving a simulator does not replace an onroad driving evaluation, it is more useful than the traditional clinical evaluation performed in daily rehabilitation practice.,

In the preliminary survey, only $35.6 \%$ of patients achieved resumption of driving. This was in accordance with a previous report that only $30 \%$ of stroke survivors are able to resume driving. ${ }^{9}$ However, because another 40 (33.9\%) patients wished to drive again, a rehabilitation program could provide the opportunity for more patients to resume driving and achieve better social reintegration. However, medical staff must outline both the risks and potential benefits of returning to driving. It is also important to caution those unable to drive safely. Patients who perform poorly on a driving simulator may be more likely to accept that they are not ready for onroad driving. Because an understanding by patients of their current strengths and limitations generally improves their willingness to accept therapeutic decisions, driving a simulator may also be useful for patients who need to abandon the idea of resuming driving. ${ }^{7}$

In this study, only the braking task was selected to evaluate driving ability. To carry out the task successfully, patients had to recognize the appearance of a truck and stop the vehicle immediately. Previously, a report assessing driving ability in stroke patients suggested that "awareness" and "reaction time" were the most important factors determining the ability to drive again. ${ }^{10}$ Therefore, we believe that the braking task was the most appropriate means of evaluation.

The results showed that patients increased their rate of successful performance of the braking task with a reduction in reaction time because of an improvement in their driving ability after repeat training with the simulator, which provided an opportunity to assess and improve driving skills.

There were gender and age differences between our stroke patients and healthy volunteers. In spite of the older age of the stroke patients, the results showed that skills improved in parallel with those in younger volunteers during training. In addition, all participants (both male and female) in both groups were experienced drivers. Thus, we believe that 
gender and age differences were not likely to have had a major impact on the results of the study.

The average Brunnstrom stage in our patients was more than 5. Although four patients had physical disabilities, with a Brunnstrom stage of the lower extremities of 3, they could walk on their own with or without a stick. Upper and lower extremity motor impairment and disrupted coordination can impact on the ability to drive safely. Because the patients had an average braking time that did not differ significantly from that of normal volunteers, they had sufficient motor ability to control the vehicle. Generally, cognitive impairments, including those affecting attention, memory, and speed of processing, may influence the ability to drive safely by impairing the ability to make quick and informed decisions. ${ }^{11,12}$ However, in this study, any mental disturbances in the patients were mild, because the mean MMSE was 27.9. Furthermore, no patient with unilateral spatial neglect, abnormalities of visual depth perception, or impaired communication ability, was included in this study. Therefore, most of the patients participating in this program had adequate mental and physical abilities to drive a car. We strongly suggest that if stroke patients with disabilities similar to our patients hope to resume driving, they can achieve their goal with adequate support and a suitable training program. However, this study provided results from a limited driving scenario. Therefore, because there are many patients who suffer from visual and other cognitive disabilities, further studies should examine other environments and evaluate other abilities.

It is important to encourage patients who can potentially resume driving to do so in order to promote community reintegration. ${ }^{13}$ The support for resumption of driving after a stroke should consist of two parts, ie, provision of adequate information about how to manage the resumption of driving and vehicle driving training using a simulator. Support for driving resumption should be continuously provided by a multidisciplinary team consisting of physicians, nurses, physical therapists, and occupational therapists, and is an essential component of effective delivery of care. ${ }^{14}$ Furthermore, team members must have a thorough knowledge of the legal and practical aspects of resuming driving, in addition to specialized stroke rehabilitation. With regard to driving

International Journal of General Medicine

\section{Publish your work in this journal}

The International Journal of General Medicine is an international, peer-reviewed open-access journal that focuses on general and internal medicine, pathogenesis, epidemiology, diagnosis, monitoring and treatment protocols. The journal is characterized by the rapid reporting of reviews, original research and clinical studies across all disease areas. training, driving simulators, such as the one used in this study, are unfortunately not available in most rehabilitation units. However, an alternative to simulator driving could be a structured driving evaluation in real traffic. The authors propose that comprehensive support is needed for stroke patients who wish to resume driving. The results of this study may be useful for medical staff who care for stroke patients and wish to enhance their social reintegration.

\section{Disclosure}

The authors report no conflict of interest in this work.

\section{References}

1. Lundqvist A, Gerdle B, Ronnberg J. Neuropsychological aspects of driving after stroke: In the simulator and on the road. Appl Cogn Psychol. $2000 ; 14: 135-150$

2. Johnson JE. Urban older adults and the forfeiture of a driver's license. J Gerontol Nurs. 1999;5:12-18.

3. Anstey KJ, Wood J, Lord S, Walker JG. Cognitive, sensory, and physical factors enabling driving safety in older adults. Clin Psychol Rev. 2005; 25:45-65.

4. Ponsford AS, Vittanen M, Lundberg C, Johansson K. Assessment of driving after stroke: A pluridisciplinary task. Accid Anal Prev. 2008;40: 452-460.

5. Perrier MJ, Korner-Bitensky N, Petzold A, Mayo N. The risk of motor vehicle crashes and traffic citations post stroke: A structured review. Top Stroke Rehabil. 2010;17:191-196.

6. Salter K, Foley N, Teasell R. Social support interventions and mood status post stroke: A review. Int J Nurs Stud. 2009;47:616-625.

7. Stern EB, Davis ES. Driving simulators. In: Pellerito JM Jr, editor. Driver Rehabilitation and Community Mobility. Principles and Practice. Philadelphia, PA: Mosby; 2006.

8. Akinwuntan AE, DeWeerdt W, Feys H, et al. Effect of simulator training on driving after stroke: A randomized controlled trial. Neurology. 2005;65:843-850.

9. Fisk GD, Owsley C, Pulley LV. Driving after stroke: Driving exposure, advice, and evaluations. Arch Phys Med Rehabil. 1997;78: $1338-1345$.

10. Schanke AK, Sundet K. Comprehensive driving assessment: Neuropsychological testing and on-road evaluation of brain injured patients. Scand J Psychol. 2000;41:113-121.

11. McCulloch K. Attention and dual-task conditions: Physical therapy implications for individuals with acquired brain injury. J Neurol Phys Ther. 2007;31:104-118.

12. Lincoln N, Majid M, Weyman N. Cognitive rehabilitation for attention deficits following stroke. Cochrane Database Syst Rev. 2000;4: CD002842.

13. Finestone HM, Guo M, O'Hara P, et al. Driving and reintegration into the community in patients after stroke. Phys Med Rehabil. 2010;2: 497-503.

14. Long AW, Kneafsey R, Ryan J. Rehabilitation practice: Challenges to effective team working. Int J Nurs Stud. 2003;40:663-673.

\section{Dovepress}

A key focus is the elucidation of disease processes and management protocols resulting in improved outcomes for the patient. The manuscript management system is completely online and includes a very quick and fair peer-review system. Visit http://www.dovepress.com/ testimonials.php to read real quotes from published authors. 